\title{
A SPECTROSCOPIC AND OPTICALLY DETECTED MAGNETIC RESONANCE STUDY OF PO=- IN POTASSIUM CHLORIDE. III. PHOTO-ORIENTATION .
}

\author{
S.J. HUNTER, K.W. HIPPS*, Richard BRAMLEY** and A.H. FRANCIS \\ Department of Chemistry, University of Michigan, Ann Arbor, Michigan 48109, USA
}

Received 10 July 1979

\begin{abstract}
The small molecular anion $\mathrm{PO}_{2}^{-}$has been incorporated at chloride ion vacancies in the potassium chloride lattice, where the relatively high symmetry of the crystal field permits a variety of crystallographically equivalent orientations of the ion within the vacancy site. Electronic excitation to the first excited singlet state of $\mathrm{PO}_{2}^{-}\left({ }^{1} \mathrm{~B}_{1}\right)$ is followed by rapid radiationless relaxation and intersystem crossing to the phosphorescent ${ }^{3} B_{1}$ state. Subsequent electronic relaxation to the ground state is found to proceed with a reorientation of the ion in the muiti-minimum crystal potential. At temperatures sufficiently low that competitive thermal reorientation of the ions is quenched, it is possible to prepare photo-oriented arrays of $\mathrm{PO}_{2}^{-}$ions in the anion vacancies by irradiation with plane polarized light at special angles. The temperature dependence of the rate of photo-orientation has been determined and it has been found possible to describe the observed kinetics in terms of a simple phenomenological rate equation. Photoorientation-evidently results from an extraordinarily strong linear electron-phonon coupling and may be used for a detailed study of this type of interaction. The potential barrier for conyersion between crystallographically equivalent sites is found to be $783 \mathrm{~cm}^{-1}$.
\end{abstract}

\section{Introduction}

An intriguing aspect of the lattice dynamics of small molecule impurity centers in alkali halide lattices is the possibility of nearly free rotation about one or more axes. In those instances where the potential barrier is small compared with $k T$, the rotational motion is thermally stimulated, as has been shown for $\mathrm{CN}^{-}$ion in $\mathrm{KCl}$ [1]. When the potential barrier to rotation is high with respect to $k T$, rotation may be stimulated by optical excitation of the impurity center as has been achieved for $\mathrm{S}_{2}^{-}$ ion in $\mathrm{KCl}$ [2]. For hydrogen bearing impurity centers such as the $\mathrm{OH}^{-}$ion in $\mathrm{KCl}$ [3] the rotational motion may involve a tunneling component and thus proceed even though $k T$ is small compared with the barrier height. A particularly dramatic

* Present address: Department of Chemistry, Washington State University, Puilman WA 99164, USA.

** On leave from Research School of Chemistry, Australian National University, Canberra, A.C.T. 2600, Australia. example of nearly free rotational motion of an impurity center is provided by $\mathrm{NO}_{2}^{-}$ion in $\mathrm{KCl}$ [4]. The vibrational and electronic spectra of this ion have been interpreted to indicate a nearly free rotation of the ion about an axis parallel to the oxygen-oxygen axis at $2 \mathrm{~K}$ [5]. Moreover, the fine structure accompanying the vibrational structure of the electronic emission and absorption spectra has been analyzed as rotational structure [6].

When the potential barrier for interconversion between crystallographically equivalent orientations is high with respect to thermal energies, the possibility still exists for photo-orientation of the impurity centers. In the photo-orientation process, photo-excitation of the impurity center is used to produce a preferential population of one particular orientation of the impurity center. It is important at this point to distinguish the photo-orientation process from photo-selection [7] or site-selection [8] processes, which do not involve a change in the 
orientation of the molecules, but rather the selection of an oriented set of molecules from a larger, unoriented set. Thus, photo-orientation refers to a dynumical process by which molecules are brought into a particular spatial arrangement, whereas photoselection is a static process in which a set of aligned molecules is selected for study. The photo-orientation process is a photo-physical process and is to be distinguished from photo-chemical "hole burning" processes. in which a selected set of molecules is caused to undergo photo-chemical decomposition by intense laser excitation. Photo-orientation has been proposed as the mechanism responsible for the bleaching of the absorption spectrum of perylene [9]- and proto-porphorin [10] in glassy solutions at $4.2 \mathrm{~K}$.

This article is the third part in a series dealing with the spectroscopic properties of potassium chloride crystals containing $\mathrm{PO}_{2}^{-}$impurity centers [11.12]. It is the purpose of the present work to describe experiments which demonstrate the photoorientation process for $\mathrm{PO}_{2}^{-}$ions assumed to be trapped at halide ion vacancies in $\mathrm{KCl}$. In the previous two parts of this work we have established the chemical nature of the impurity center and the orientation of the molecular axes of the excited ${ }^{3} \mathbf{B}_{1}$ state with respect to the cubic crystallographic axes.

\section{Experimental}

The method of preparation of $\mathrm{KCl}$ crystals containing $\mathrm{PO}_{2}^{-}$impurity centers has been described previously [11]. Crystals were cleaved parallel to the principal cleavage planes of the cubic lattice $\{100\}$ to approximate dimensions $3 \mathrm{~mm} \times 3 \mathrm{~mm} \times 5 \mathrm{~mm}$ and mounted on a sample holder milled from solid brass. Graphite filled silicone grease was used to provide good thermal coniact between sample and holder.

For these experiments, precise temperature control and measurement were essential, and considerable care was taken in the design of a temperature control system. Two levels of temperature regulation were required to achieve the desired temperature stability. Liquid helium from a $4.2 \mathrm{~K}$ bath was vaporized by a resistively heated copper diffuser assembly. the temperature of which was regulated by a rate/proportional controller (Artronix Model 5301) to within 0.1 K over a 10-200 $K$ range. The temperature of the diffuser assembly was monitored with a Micro-Measurcments cryogenic linear temperature sensor, Model CLTS-2, which was epoxied to the copper diffuser block. Precise control of the temperature at the sample was achieved with an additional rate/proportional controller (Artronix Model 5301) which regulated the temperature of the resistively heated sample block. The temperature of the sample biock was measured using a calibrated Scientific Instruments Model N2G four-lead germanium resistance thermometer embedded in graphite filled silicone grease in a hole located $1 \mathrm{~mm}$ behind the sample position. This system made it possible to maintain temperatures at the sample constant to within $\pm 0.01 \mathrm{~K}$ at $10 \mathrm{~K}$ or $\pm 0.05 \mathrm{~K}$ above $40 \mathrm{~K}$ for an hour or longer.

The time dependence of $\mathrm{PO}_{2}^{-}$phosphorescence was investigated as a function both of temperature and of the polarization and intensity of the ultraviolet excitation radiation. The excitation source was a I KW high pressure mercury-xenon lamp, the output of which was filtered by a combination of an aqueous $\mathrm{Ni} / \mathrm{CoSO}_{4}$ solution and Corning 7-54 glass filter. This combination of filters provided a bandpass of approximately $800 \AA$ centered about $2950 \AA$. The polarizer and analyzer were both $5 \mathrm{~cm}$ diameter $3 \mathrm{M}$ Co. Model PL-40 polarizing filters, which provided approximately $97 \%$ linearly polarized light between $6000-2500 \AA$ and had an average transmittance of approximately $35 \%$.

\section{Results}

The kinetics of photo-orientation were investigated by following the time evolution of phosphorescence intensity as a function of the polarization and intensity of the incident radiation for several different temperatures of the sample. For a fixed crystal temperature, the incident radiation was plane polarized vertically $\left(P_{\mathbf{y}}\right)$ and was parallel to a cubic [100] axis. The phosphorescence intensity was monitored through a horizontal analyzer $\left(A_{11}\right)$ at $90^{\circ}$ to the direction of excitation and was recorded as a function of time until a constant level of intensity was obtained. The time required to obtain a steady- 
state phosphorescence level was linearly dependent on the incident radiation intensity and was typically on the order of $10 \mathrm{~min}$ for an incident light intensity. of $0.3 \mathrm{~W} / \mathrm{cm}^{2}$.

After equilibrium levels of phosphorescence intensity had been obtained with vertically polarized incident radiation, the polarizer was rotated to the horizontal $\left(P_{\mathrm{H}}\right)$, resulting in an immediate increase in the level of phosphorescence followed by decay to a new steady-state level. The polarizer was then rotated to the vertical position and the decay to the original steady-state phosphorescence level was recorded. The sample temperature was then changed and the series of measurements repeated. The time dëperidence of the phosphorescence intensity for the series of operations described above is illustrated in fig. 1 for a range of sample temperatures. The time dependence of the phosphorescence intensity illustrated in fig. 1 was found to be very nearly exponential over the temperature range $30-45 \mathrm{~K}$. It was possible to obtain a linear plot of the logarithm of phosphorescence intensity versus time (in [I] versus $t$ ) for each decay illustrated in fig. 1. A family of curves is reproduced in lig. 2. The slope of each curve corresponds to the rate constant for photoorientation at the temperature indicated. It is clear that the rate constant for photo-orientation approaches a limiting value ( $\left.\ln k_{0}\right)$ as the tempera-

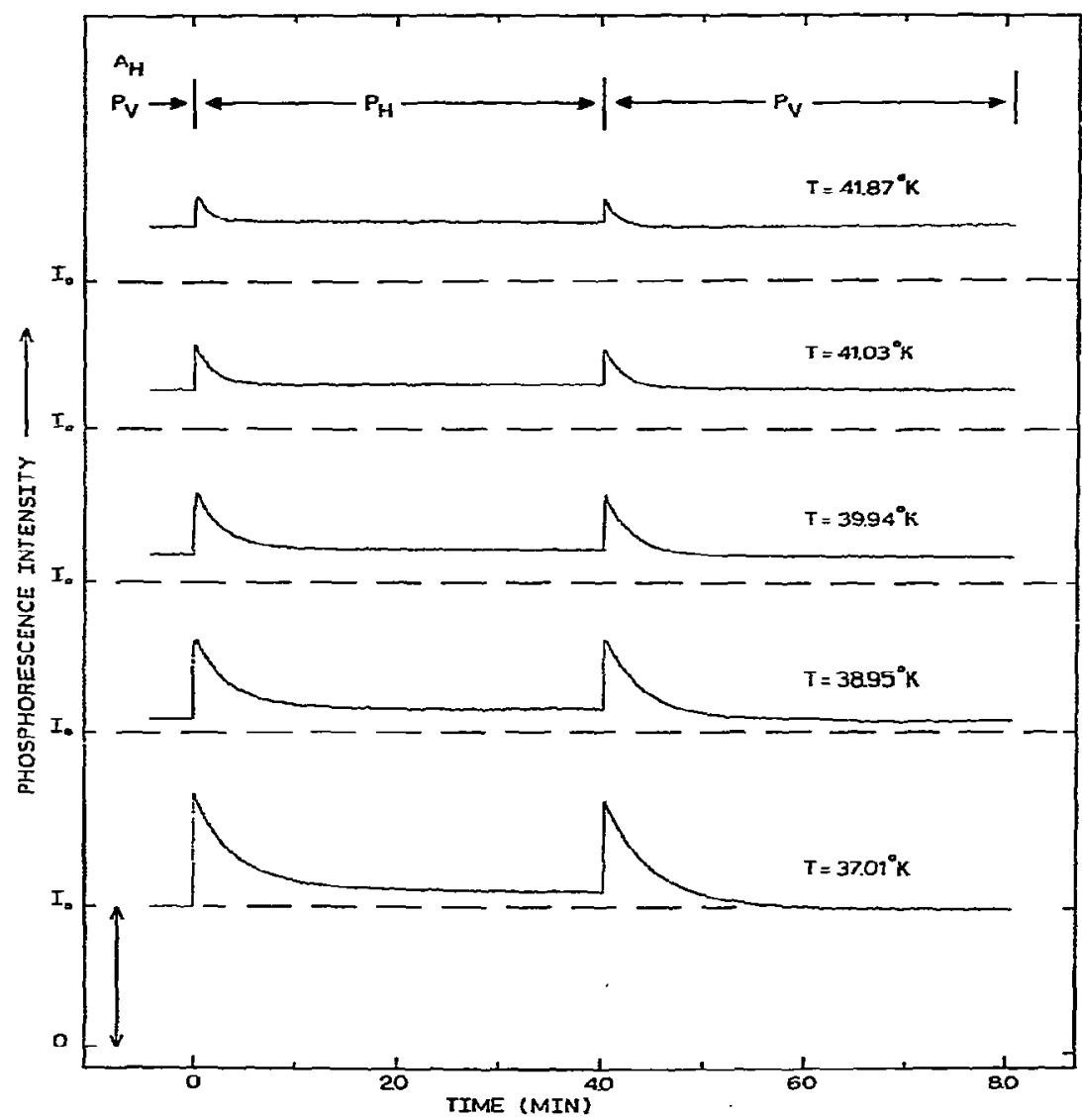

Fig. 1. Time dependence of the phosphorescence intensity observed for several sample temperatures and two polarizer positions. 


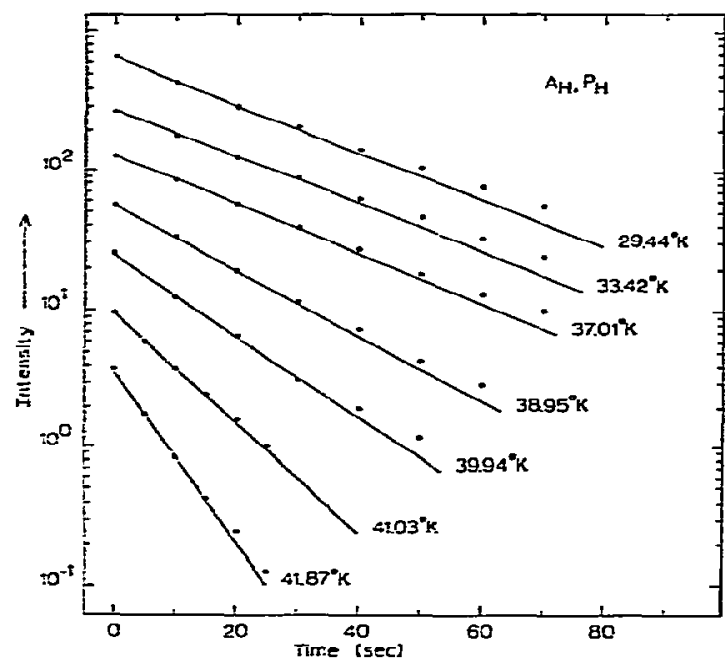

Fig- 2. Semi-loæ plot of phosphosrescence intensity versus time for several sample temperatures.

ture decreases. This is most evident from the plot of the temperature dependent rate constant versus $1 / T$ shown in fig. 3.

The following observations concerning the time dependence of the phosphorescence intensity over the temperature range $30-45 \mathrm{~K}$ were made:

(I) For sample temperatures above approximately $45 \mathrm{~K}$. the phosphorescence intensity is independent of incident polarization and does not evolve in time.

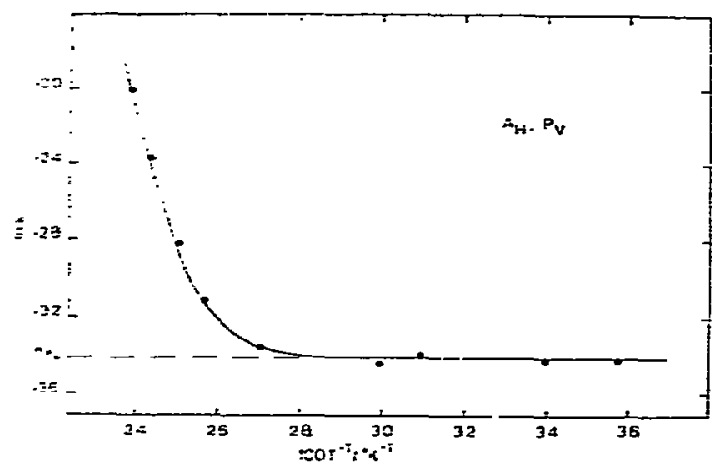

Fig. 3. Semi-log plot of the photo-orientation rate constant versus $1 / T$. illustrating the tempcrature dependent and temperature independent portions of the total rate constint
(2) Between $35 \mathrm{~K}$ and $45 \mathrm{~K}$, both the change and rate of change of phosphorescence intensity following a $90^{2}$ rotation in incident light polarization are strongly temperature dependent.

(3) Between $30 \mathrm{~K}$ and $35 \mathrm{~K}$, the sample phosphorescence intensity is both poiarization and time dependent but the decay kinetics are independent of temperature.

(4) At all temperatures, the asymptotic levels of phosphorescence intensity are indefinitely reproducible.

The decay curves shown in fig. 1 and their temperature behavior illustrated in figs. 2 and 3 clearly indicate the presence of a rate constant which contains both a temperature dependent and a temperature independent component. The temperature independent component was determined from the low temperature curves where the rate constant asymptotically approaches the limiting value $\ln \left(k_{0}\right)$, as shown in fig. 3 . The limiting rate constant $\left(k_{0}\right)$ was subtracted from the total rate constant $(k)$ at higher temperatures to give a set of rate parameters which was characteristic of the temperature dependent process alone. These fitted an Arrhenius plot within experimental error (fig. 4) and gave a frequency factor of $0.6 \times 10^{11} \mathrm{~s}^{-1}$ and an activation energy of $783 \mathrm{~cm}^{-1}$.

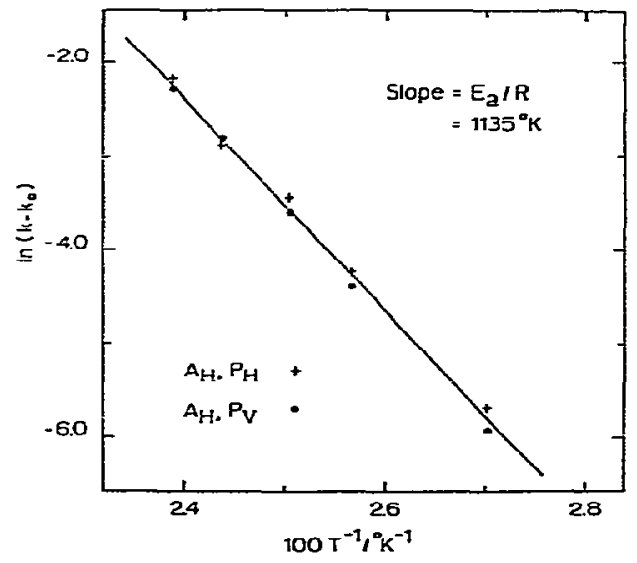

Fig. 4. Semi-log plot of the temperature dependent portion of the photo-orientation rate constant versus $1 / T$, illustrating a single activation cnergy for the process. 


\section{Discussion}

\subsection{Kinetic model}

The reproducibility of the equilibrium levels of phosphorescence intensity for the same set of experimental conditions indicates that the time evolution of the phosphorescence intensity is not due to photo-chemical decomposition of the emitting species. For this reason, a model based upon the reorientation of the emitting species between crystallographically equivalent sites by plane polarized excitation was developed to interpret the temporal behaviour of the phosphorescence intensity. This model is capable of rationalizing the observed kinetics in quantitative detail, utilizing parameters established from independent experiments.

We have previously established the orientation of the excited triplet state of the $\mathrm{PO}_{2}^{-}$ion in the chloride ion vacancy of potassium chloride by optical and magnetic resonance measurements. Using the conventional molecular axis designation with $z$ parallel to the two-fold axis of the $C_{2 v}$ ion and $x$ perpendicular to the molecular plane, we have determined that the ions occupy positions in which the molecular $z$ axis is directed parallel to the cubic axes [100] and the molecular $x$ and $y$ axes are

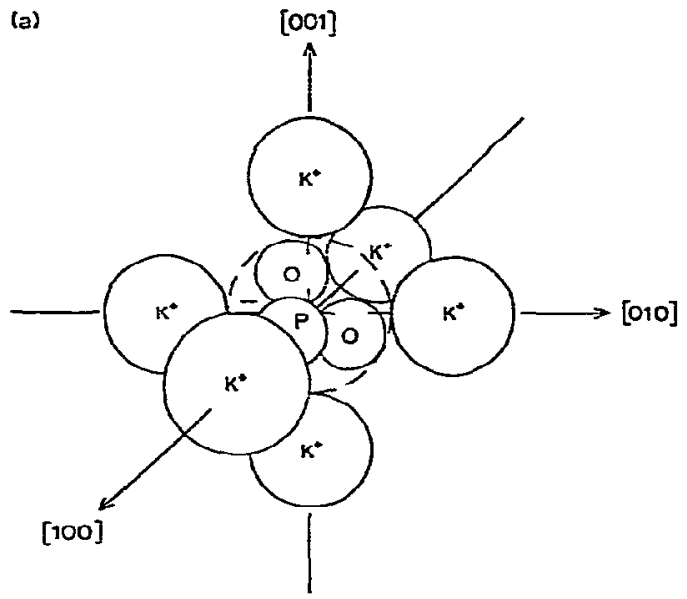

directed along the $[110]$ dircctions. There are, accordingly, twelve crystallographically equivalent orientations of the $\mathrm{PO}_{2}^{-}$ion in the halide ion vacancy, one of which is illustrated in fig. 5a. These twelve orientations belong to one of three sets, 1,2 , and 3 , according to the planes which contain the $x$ and $y$ axes, $\{010\},\{001\}$, and $\{100\}$, respectively.

Experiments were conducted with incident radiation plane polarized parallel to the [ 100$]$ crystallographic axes and, since the optical excitation cross section depends only upon the squared projection of the radiation electric field vector upon the molecular electric dipole transition moment, the four crystallographically equivalent molecules of each set are indistinguishable in the optical experiment.

In the absence of incident radiation, all twelve crystallographically equivalent orientations are equally probable. It was assumed that interconversion occurs only between orientations related by $90^{\circ}$ rotations of the ion about $[100],[010]$ and $[001]$ axes as illustrated in fig. $5 \mathrm{~b}$. It is important to note that interconversion need not occur by a simple $90^{\circ}$ rotation about these axes.

There are three inequivalent and distinguishable photo-excitation rate constants, one for each optically distinguishable set. The photo-excitation

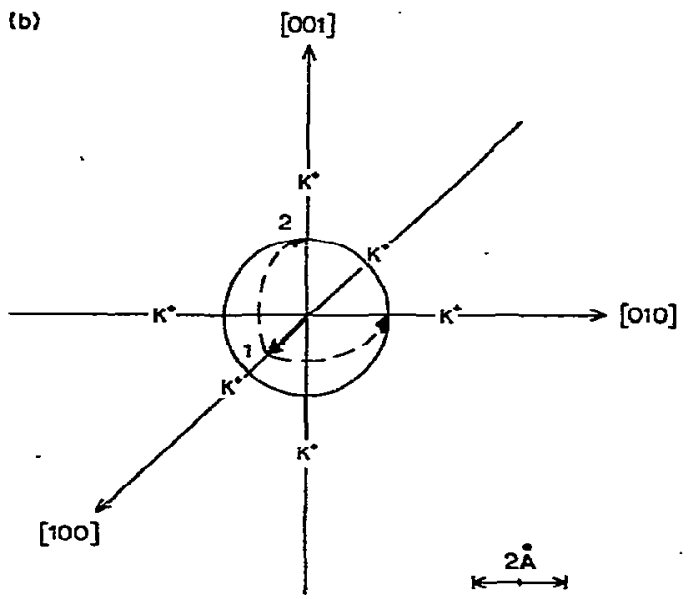

Fig. 5. The orientation of the $\mathrm{PO}_{2}^{-}$ion in the ${ }^{3} \mathrm{~B}_{1}$ state. There are 12 crystallographically equivalent positions, interconverted by $90^{\circ}$ rotations as illustrated. 
rate constant for an ion of the ith set may be written as:

$$
k_{i}=\left|\mu_{\mathrm{s}} \cdot M_{i}^{\prime \prime} \cdot E_{\mathrm{p}}\right|^{2} \text {, }
$$

where $\mu_{\Lambda}$ is the absorption transition electric dipole moment in the molecular axis system defined above. The orientation of the electric field vector $\left(E_{\mathrm{p}}\right)$ is determined by the setting of the polarizer and is referred to the crystallographic axis system. $\mathbf{M}_{i z}^{\prime \prime}$ is the matrix of the direction cosines for a ground state ion of the ith set with respect to the crystallographic axes.

The rate equations for reorientation were developed from the kinetic scheme illustrated in fig. 6. We separate the rate constant for vibronic relaxation into a rate constant for relaxation with reorientation in the lattice $(k)$ and a rate constant for relaxation without reorientation $(\bar{k})$. Additionally, we assume that the rate constant for thermal reequilibration in the ground electronic state may be written as

$k^{*}=A \exp [-v(0) / k T]$.

where $A$ is a constant independent of temperature and $V(0)$ is the effective potential barrier to

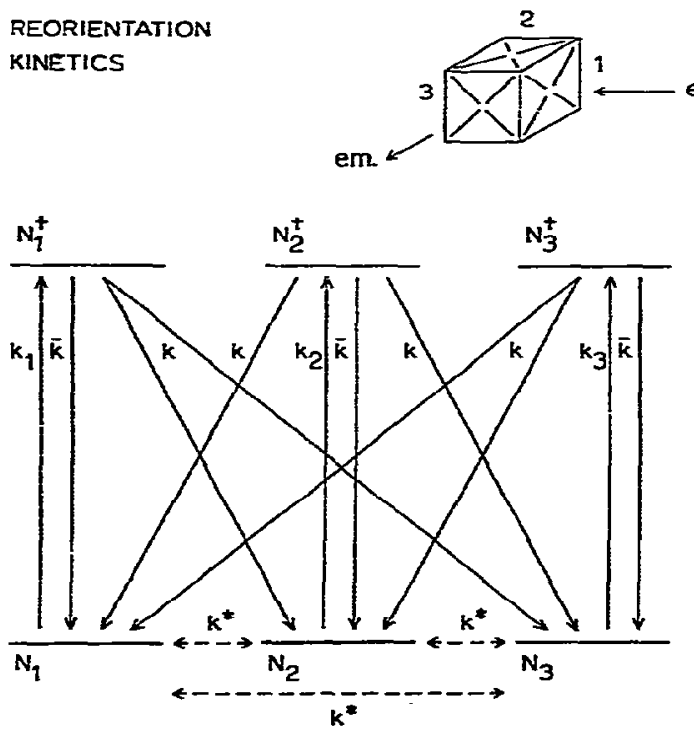

Fig 6. The kinetic model used to develop the photoorientation kinetic equations. reorientation between sites related by a $90^{\circ}$ rotation. With these assumptions, we obtain the following differential rate equation for the population of the ith set:

$$
\mathrm{d} N_{i} / \mathrm{d} t=-2 N_{i} K_{i}+\sum_{j \neq i} N_{j} K_{j},
$$

where $K_{i}=\left(k k_{i}+k^{*} \bar{k}+2 k k^{*}\right) /(\bar{k}+2 k)$ and the summation extends over the remaining sets. We have employed the steady-state approximation with respect to the photoexcited populations $\left(N_{i}^{t}\right)$ in order to obtain eq. (3).

Taking the Laplace transform of eq. (3), we obtain:

$n_{i}(p)-N_{i}^{0}=-2 K_{i} n_{i}+\sum_{j \neq i} K_{i} n_{j}$,

where $N_{i}^{\mathrm{e}}$ is the population of the ith set at time $t=0$. the start of irradiation, and $n_{i}(p)=\mathscr{L}\left\{N_{i}(t)\right\}$. The $n_{i}(p)$ may be obtained from the solution of the set of linear equations (4) as ratios of the form:

$n_{\mathrm{i}}(p)=H_{\mathrm{i}}(p) / D(p)$,

where $H_{i}(p)$ and $D(p)$ are polynomials in the Laplace variable $p . D(p)$ is the characteristic equation of the matrix of the coefficients of the $n_{i}(p)$ in eq. (4). $H_{i}(p)$ is the characteristic equation of the matrix with the ith column replaced by the initial populations. Employing the Heavyside expansion theorem, we obtain the time dependent populations of the optically inequivalent sets as:

$N_{k}(t)=\sum_{k} \frac{H_{i}\left(p_{k}\right)}{D^{\prime}\left(p_{k}\right)} \exp \left(p_{k} t\right)$

where the $p_{k}$ are the roots of $D(p)$ of which all are real and $D^{\prime}(p)=\mathrm{d} D / \mathrm{d} p$. The summation extends over all roots $p_{k}$. Finally, the time dependence of the emission intensity is obtained by squaring the projection of the emission transition dipole moment on the polarization vector of the analyzer and summing over all optically inequivalent sets. We obtain:

$$
\begin{aligned}
& I(t)=\sum_{i} \sum_{k} \frac{I_{i}\left(p_{k}\right)}{D^{\prime}\left(p_{k}\right)}\left|\mu_{A} \cdot M_{i}^{\prime \prime} \cdot E_{\mathrm{p}}\right|^{2}\left|\mu_{E}-M_{i}^{\prime}-\pi_{A}\right|^{2} \\
& \quad \times \exp \left(p_{k} t\right) .
\end{aligned}
$$

where $\pi_{A}$ is the polarization vector of the analyzer and $\boldsymbol{\mu}_{\mathrm{E}}$ is the emission transition dipole moment. $\mathbf{M}_{\overline{\boldsymbol{i}}}^{\prime}$ is the matrix of the direction cosines for an ion of 
the ith excited state set. The elements of this matrix were determined in part $I$ of this work.

The optical excitation bandwidth was restricted to the range $2500-3400 \AA$ so that the direction of the absorption dipole moment is entirely $\mu_{x}\left({ }^{2} B_{1} \leftarrow{ }^{1} A_{1}\right)$. Emission is principally polarized parallel to the $y$ molecular axis with a small component parallel to $z$ $[11,12]$

$\mu_{\mathrm{E}}=\mu_{\boldsymbol{y}} \dot{j}+\mu_{z} k$.

The magnitudes of $\mu_{E}$ and $\mu_{A}$ may be established within rather narrow limits by separate experimental measurements. Eq. (7) then contains only $A$, the Arrhenius prefactor, $V(\theta)$ the potential barrier to reorientation and the ratio $k / \bar{k}$, to be determined from the observed kinetics of photo-orientation.

\subsection{Ion orientation}

In order to apply eq. (7) to the experimental kinetic data, it was necessary to make certain initial assumptions about the orientation of the $\mathrm{PO}_{2}^{-}$ion in the ground state (i.e., the elements of $\mathbf{M}_{i}^{\prime \prime}$ ). Initially it was assumed that the equilibrium ground state orientation was identical to that observed for the ${ }^{3} B_{1}$ state, however, it proved impossible to duplicate several salient features of the experimental data with this assumption. The principal deviation between the observed and the calculated kinetic behavior occurred in the asymptotic limit approached at long irradiation times. Typically the asymptotic limit is $45 \%$ of the initial phosphorescence intensity, while the theoretical model predicts that in the asymptotic limit the phosphorescence intensity should approach zero. The observed behavior requires either a component of $z$ polarized absorption or a rotation of the ion about its $y$-axis in the ground state. A third possibility, that some of the $\mathrm{PO}_{2}^{-}$ions are statistically disoriented by random strains or clustering was also considered. However, it was possible to apply a weak ( $300 \mathrm{G}$ ) magnetic field to remove the orientational degeneracy in the optically detected magnetic resonance (ODMR) spectrum. Then, photo-orientation experiments were repeated on several components of the ODMR spectium, each of which corresponds to a set of ions uniformly oriented with respect to the magnetic held. The photo-orientation kinetics observed for each set of ions studied was identical in its asymptotic behavior to that observed for the bulk sample emission and we conclude that this behavior is not die to the inclusion of misoriented ions.

The asymptotic behavior is most easily attributed to the presence of some $z$-polarized absorption. Although this is not possible for the ${ }^{1} B_{1} \leftarrow{ }^{1} A_{1}$ transition due to the absence of $a b_{2}$ molecular vibration, the $z$-polarized ${ }^{1} A_{2} \leftarrow{ }^{1} A_{1}$ transition may also be excited within the bandpass used. In order to examine this possibility, several photo-orientation experiments were performed using narrow bandwidth $(50 \AA)$ excitation between 2400 and $3000 \AA$. It was anticipated that selective excitation of the $x$-polarized ${ }^{1} B_{1} \leftarrow{ }^{1} A_{1}$ transition or the z-polarized ${ }^{1} A_{2} \leftarrow{ }^{\prime} A_{1}$ transition would produce dramatically different asymptotic behavior of the photo-orientation kinetics. It was determined that the asymptotic behavior was completely independent of the wavelength within the excitation bandpass used in our experiments. Evidently no significant $z$-polarized absorption is present within this bandpass.

We conclude, therefore, that the ion is in fact rotated in the ground state from the equilibrium position determined for the ${ }^{3} \mathrm{~B}_{1}$ state. Rotation about the $y$-axis is required to bring the experimental kinetic behavior and that predicted by eq- (7) into agreement. The amount of rotation required may be obtained from eq. (7) in the asymptotic limit and is found to be $35^{\circ}$.

\subsection{Reorientation model}

Several models which may be employed to explain the observed photo-orientation of the $\mathrm{PO}_{2}^{-}$color centers in $\mathrm{KCl}$ will now be discussed briefly. The first involves photo-orientation in the excited state according to a model originally proposed for site conversion in rigid organic glasses. The observation of non-photochemical hole burning in organic glasses [13] has been explained, in part, by postulating site conversion within an inhomogeneously broadened linewidth under the action of optical excitation usually provided by intense laser irradiation. A "two site" model for the process suggested by Hayes and Small [14], involves the reorientation of solute molecules by displacement along a double minimum potential coordinate. The exact nature of 
the coordinate is usually undetermined, but it may correspond to a rotation or translation of the solute molecule. Within the framework of the model, a vitreous matrix site is described by a double minimum potential and the matrix as a whole is composed of a set of such sites with different barriers between minima and different coordinate descriptions. Reorientation is postulated to occur in the excited state for a subset of sites which have a low barrier for reorientation of the excited solute molecule. Solute molecules in these sites have a finite probability of reorienting in the excited state within their radiative lifetime and, upon subsequent radiative relaxation, returning to new ground state equilibrium orientations. In the relatively low site symmetry of a vitreous material, the newly occupied site will with high probability, have a slightly different excitation energy than the original site and therefore no longer be in resonance with the narrow band laser excitation source. By this mechanism a (a)

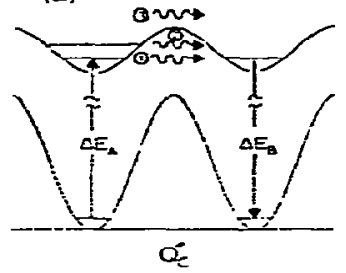

a

B

(c)

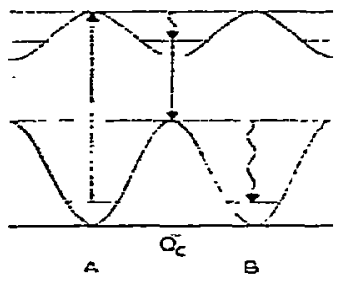

(b)

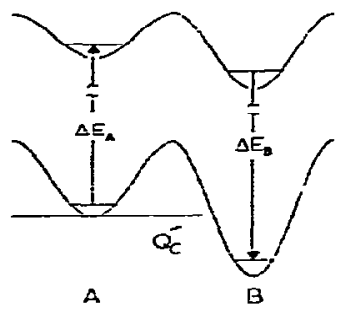

(d)

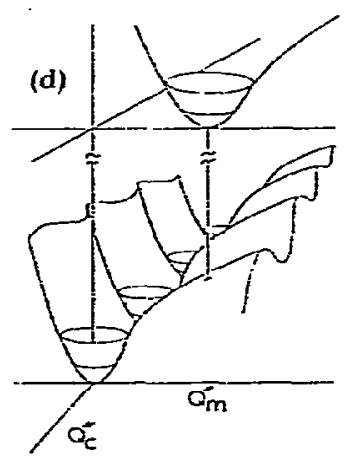

Fig 7. Some possible relationships between ground state and excited state potential surfaces along a lattice configuration coordinate $\underline{Q}_{c}$ and a selected molecular normal coordinate $Q_{\mathrm{m}}$. "hole" is burned in the inhomogeneously broadened absorption spectrum. The relationship between the ground state and excited state potential surfaces in the "two site" wodel is illustrated in fig. $7 \mathrm{~b}$.

Although the potential surface in a crystalline lattice is substantially different than that in vitreous matrices, the model proposed by Hayes and Small may still be utilized in part. The principal difference between the vitreous solvent and the crystalline site is the degree of symmetry of the crystal potential hypersurface. In the case of the anion site in alkali halide crystals, the site has two fold symmetry about [110] and four fold symmetry about [100] axes. The site conversion in high site symmetries involves, principally, reorientation between energetically equivalent but orientationally distinguishable sites (fig. 7a). Thus, in the vitreous matrix, reorientation proceeds with both a change in the absorption frequency and polarization, while in the crystalline lattice only a change in absorption polarization may occur.

A second model may be developed in which the photo-orientation step occurs in the ground state. The ions $S_{2}^{-}[15], \mathrm{O}_{2}^{-}[15]$ and $\mathrm{NO}_{2}^{-}[16]$, which have also been shown to photo-reorient in some alkali halide lattices, have a spectral characteristic in common with $\mathrm{PO}_{2}^{-}$. Upon excitation to the low lying electronic excited states, large displacements along internal normal coordinates occur and the spectra of these ions characteristically exhibit long progressions in stretching and bending modes. As a consequence of the large displacements in molecular coordinates, vertical electronic relaxation from the lowest triplet state terminates with maximum probability high on the ground state potential surface along the displacement coordinate. The vibrational excitation energy is sufficient to thermally activate site conversion, however, a relatively strong coupling between the lattice displacement coordinate and the vibrationally excited internal mode is also required for efficient site conversion. We note that the extraordinarily intense multiphonon sidebands exhibited by the spectra of many ions which photo-orient in alkali halide lattices is an indication of strong linear electrori-phonon coupling. The relationship between the molecular and crystal potential surfaces required for photoorientation by this mechanism is illustrated in fig. $7 \mathrm{~d}$. 
A third model, invokes partial reorientation in both the ground and excited state. In the case of $\mathrm{PO}_{2}^{-}$, the ground state equilibrium orientation has been shown to be substantially altercd from that of the ${ }^{3} \mathrm{~B}_{1}$ state by a $35^{\circ}$ rotation about the molecular $y$-axis. We may imagine a suitable configuration coordinate $\left(Q_{d}\right)$ for the rotation which interconverts equivalent sites. If the excited state potential hypersurface is sufficiently displaced along this lattice coordinate, reorientation may occur as illustrated in fig. 7c.

At present it is not possible to determine the exact mechanism for photo-orientation from the available experimental data. However, a dramatic decrease in the photo-orientation rate at temperatures below $30 \mathrm{~K}$ indicates that the photo-orientation step is partially thermally assisted. Hayes and Small [14] have suggested that site conversion in the "two site" model may proceed at elevated temperatures by a thermally activated mechanism and at low temperatures by a phonon assisted tunneling mechanism. Qualitatively, this suggestion is in agreement with our observations and favors excited state photo-orientation.

Finally, we note that several local minima exist in the ground state potential surface in which ions may become "trapped" in metastable orientations at sufficiently low temperatures. If the cross section for photo-excitation of such metastable orientations is small, this would also result in a decrease in the photo-orientation rate with decreasing temperature. Work is presently in progress on the temperature dependence of the photo-orientation rate at temperatures below $30 \mathrm{~K}$ and on the calculation of the crystal potential using simple pairwise repulsive interactions in an attempt to further elucidate the photo-orientation mechanism.

\section{References}

[1] W.D. Seward and V. Narayanamurti, Phys. Rev. 148 (1966) 463.

[2] L.A. Rebane, A.B. Treshchalov and T.Yu. Khal'dre, Soviet Phys. Solid State 16 (1975) 1460.

[3] F. Luty, Phys. Rev. BI0 (1974) 3667.

[4] V. Narayanamurti, W.D. Seward and R.O. Pohl, Phys. Rev. 148 (1966) 481.

[5] R. Avarmaa and L.A. Rebane, Phys. Stat. Sol. 35 (1969) 107.

[6] T.J. Haldre, L.A. Rebane, A.V. Liapzev and A.A. Kiselev, Phys. Stat. Sol. B70 (1975) 359.

[7] A.C. Albrecht, J. Mol. Spectry. 6 (1961) 84.

[8] A.N.Anoshin, E.A. Gastilovich, G.T. Kryuchkova, B.K. Sokolov, D.N. Shigonin and F.Ya. Frolov, Opt. Spectry. 44 (1968) 61.

[9] R.I. Personov, E.I. Al'shitz and L.A. Bykovskaya, JETP Letters 15 (1972) 431.

[10] S. Voelker, R.M. Macfariane, A.Z. Genack, H.P. Trommsdorff and J.H. van der Waals. J. Chem. Phys. 67 (1977) 1759.

[11] S.J. Hunter, K.W. Hipps and A.H. Francis, Chem. Phys. 39 (1979) 209.

[12] S.J. Hunter, K.W. Hipps and A.H. Francis, Chem. Phys. 40 (1979) 367.

[13] J.M. Hayes and G.J. Small, Chem. Phys. Letters 54 (1978) 435.

[14] J.M. Hayes and G.J. Small, Chem. Phys. 27 (1978) 151.

[15] L.A. Rebane and A.B. Treshchalov, J. Luminescence, $12 / 13(1976) 425$.

[16] A.M. Freiberg, A.B. Treshchalov and O.I. Sild. Zh. Prikl. Spektrosk. 28 (1978) 808. 